\title{
Impact of Renal Transplantation on Psychosocial Status of Human Immuno Deficiency Virus (HIV) Positive Patients
}

\author{
DK Agarwal*, Aditya Agarwal, Nalin Nag, Swapnil Y Gajway and Satyabrat Garanayak
}

Departments of Nephrology, Indraprastha Apollo Hospitals, New Delhi, India

\begin{abstract}
Background and Methods: Nephropathy is a common complication amongst the HIV positive population. With the advent of Combined Anti-Retroviral Therapy (cART), CD4+ counts and viral replication can be controlled more efficiently. The survival rates are fast approaching that of the general population. In such a scenario, renal transplantation is growing increasingly popular as a treatment modality of End Stage Renal Disease (ESRD) in HIV positive patients.
\end{abstract}

We studied the psychosocial changes in a subset of such HIV positive renal transplant recipients through a questionnaire which assessed multiple parameters with fixed multiple choice responses. An attempt was made to compare the pre and post-transplant psychosocial status of the patients wherever possible. We selected ten HIV positive patients who had undergone renal transplantation at our center at least one year prior to the study.

Results: Nine out of ten patients $(90 \%)$ reported better quality of life, an increase in professional productivity and perceived an improvement in behavior of the spouse and colleagues towards them. Out of nine patients who were sexually active, seven (77\%) experienced an improvement in their sexual relationship. However, seven (70\%) patients recalled that they underwent substantial anxiety and feared an unsuccessful transplant and the relapse of HIV prior to the surgery, while six $(60 \%)$ answered that they live in constant fear of an HIV relapse even after the surgery. According to all of them, the prominent factors limiting renal transplantation in HIV positive patients were substantial cost and lack of awareness of kidney transplantation as a possible option for them.

Conclusion: Renal Transplantation had a definite positive impact on the psychosocial status of HIV positive renal transplant recipients, enhancing multiple facets like quality of life, sexual relationships and professional life amongst others. Although it was a source of fear and anxiety for some. A larger study with more patients would definitely be more illuminating.

Keywords: Renal transplantation; cART; HIV positive patients; Psychosocial status

\section{Introduction}

In addition to the destroyed immune system and resulting multiple serious afflictions, HIV infection also carries with it substantial stigma, whether it be social or psychological. Every illness experienced represents a unique and dramatic negative experience for the patient; it is associated with a profound psychological engagement of the patients themselves and the significant people in their lives [1].

Combined antiretroviral therapy (cART) has transformed human immunodeficiency virus (HIV) infection into a chronic manageable infective condition since the late 90 s. Nearly $4-7 \%$ of HIV positive patients demonstrate ESRD (end stage renal disease) and ESRD has become one of the most important causes of morbidity and mortality in HIV positive patients [2]. Patients infected with HIV require renal replacement therapy (RRT) once they reach ESRD. Though in the era of cART, overall survival and morbidity of HIV Positive patients on dialysis has improved, it is still far less than patients with no renal problems and not on dialysis programs [3]. Prior to the era of potent antiretroviral therapy, the survival of HIV infected patients was too poor to justify transplantation and until recently, HIV infection was considered as an absolute contraindication to kidney transplantation [4,5]. But now, with the availability of better immunosuppressive drugs, and more experience of managing transplant patients, renal transplantation is successfully being carried out in HIV positive patients, with results at par with those of non HIV patients. HIV patients with ESRD on maintenance haemodialysis (MHD) have poor CD4+ recovery on cART as compared to those who received a renal transplant [6]. They receive haemodialysis in isolation with single use dialyzer that adds significant cost to their treatment, increasing their stress substantially. Various retrospective analyses, case reports and small prospective studies showed good patient and graft survival in HIV infected renal transplant recipients [7-9].

Currently many centres are performing successful renal transplantation in HIV infected patients. However, management of HIV infected patients during and post-transplant is still a very complex and challenging task due to multiple drug interactions, risk of HIV relapse and associated co-infections. Co-ordination between transplant and HIV physician is the key to a successful renal transplant in a HIV infected patient. However, data about the impact of renal transplantation on psychosocial status in HIV positive patients is not available. We have carried out renal transplantation of multiple HIV positive patients at our tertiary care superspeciality hospital (Indraprastha Apollo Hospitals, New Delhi, India) successfully. We

*Corresponding author: Dr. DK Agarwal, Senior Consultant Nephrology, B-109 Alpha-1, Greater Noida, Gautam Buddha Nagar, Uttar Pradesh, India, PIN-201310, Tel: +919811200113; E-mail: drdkagarwalindia@gmail.com

Received January 05, 2015; Accepted March 03, 2016; Published March 11 2016

Citation: Agarwal DK, Agarwal A, Nag N, Gajway SY, Garanayak S (2016) Impact of Renal Transplantation on Psychosocial Status of Human Immuno Deficiency Virus (HIV) Positive Patients. J AIDS Clin Res 7: 554. doi:10.4172/2155-6113.1000554

Copyright: (c) 2016 Agarwal DK, et al. This is an open-access article distributed under the terms of the Creative Commons Attribution License, which permits unrestricted use, distribution, and reproduction in any medium, provided the original author and source are credited. 
studied the impact of renal transplantation on the psychosocial status through a questionnaire which assessed multiple parameters with fixed multiple choice responses. An attempt was made to compare the pre and post-transplant psychosocial status of the patients wherever possible.

\section{Patients and Methods}

Till date, successful renal transplants of sixteen HIV Positive patients with ESRD have been performed by us at our centre (Indraprastha Apollo Hospitals, New Delhi, India). Out of them, we selected ten

\begin{tabular}{|c|c|}
\hline & $\mathrm{N}=10$ \\
\hline 1. Age (years), median (IQR) & $42.5(40,53)$ \\
\hline 2. Male, $N(\%)$ & $9(90 \%)$ \\
\hline 3. Black ethnicity, $\mathrm{N}(\%)$ & $9(90 \%)$ \\
\hline 4. Time since HIV diagnosis (year), median (IQR)4 $(3,6)$ & \\
\hline 5. CD4 cell count (cells/mm3), median (IQR) At RT409 $(348,416)$ & \\
\hline 6. HIV RNA PCR not detectable, N(\%) & $10(100 \%)$ \\
\hline \multicolumn{2}{|l|}{ 7. Viral hepatitis, $\mathrm{N}(\%)$} \\
\hline HBsAg positive & $0(0 \%)$ \\
\hline HCV Ab / HCV RNA positive & $1(10 \%)$ \\
\hline \multicolumn{2}{|l|}{ 8. Class of drugs in cART regimen, $\mathrm{N}(\%)$} \\
\hline a. 2 NRTI \& 1 NNRTI & $7(70 \%)$ \\
\hline \multicolumn{2}{|l|}{ b. 2 NRTI \& 1 PI1 (10\%) } \\
\hline c. $1 \mathrm{NRTI}, 1 \mathrm{NNRTI}, 1 \mathrm{PI}$ & $1(10 \%)$ \\
\hline d. 2 NRTI \& 1 Integrase inhibitor & $1(10 \%)$ \\
\hline \multicolumn{2}{|l|}{ 9. Time on cART (years), median (IQR) $3.5(2.5,5)$} \\
\hline \multicolumn{2}{|l|}{ 10. Dialysis } \\
\hline Haemodialysis N (\%) & $9(90 \%)$ \\
\hline Peritoneal dialysis) N (\%) & $1(10 \%)$ \\
\hline Dialysis duration (months), median (IQR) & $6.5(4,18)$ \\
\hline \multicolumn{2}{|l|}{ 11. Native kidney disease, $\mathrm{N}(\%)$} \\
\hline a. HIVAN & $2(20 \%)$ \\
\hline b. CGN & $1(10 \%)$ \\
\hline c. $\mathrm{CIN}$ & $2(20 \%)$ \\
\hline d. DN & $2(20 \%)$ \\
\hline e. HTN & $3(30 \%)$ \\
\hline
\end{tabular}

RT: Renal Transplant; HIV: Human Immunodeficiency Virus; HBsAg: Hepatitis B Surface Antigen; NRTI: Nucleoside Reverse Transcriptase Inhibitor; HCV: Ab-Hepatitis C Antibody; cART: Combination Antiretroviral Therapy; NNRTI: Non- Nucleoside Reverse Transcriptase Inhibitor; PI: Protease Inhibitor; HIVAN: HIV Associated Nephropathy; CGN: Chronic Glomerulonephritis; CIN: Chronic Interstitial Nephritis; DN: Diabetic Nephropathy; HTN: Hypertension. patients for this study who underwent renal transplantation at least one year prior to the study (Table 1). One patient was lost to follow up while five patients had not completed one year post transplantation at the time of this study. Majority of them were male (90\%), of black ethnicity (90\%), on dialysis (haemodialysis or peritoneal dialysis) with median duration of 6.5 months. At the time of transplantation, their CD4+ counts were $>200$ cells/mm3 and HIV RNA levels were not detectable while on cART therapy (median duration 3.5 years). Most patients ( 7 out of the 10) were on a combination of 2 NRTI (Nucleoside Reverse Transcriptase Inhibitor) and 1 NNRTI (Non-Nucleoside Reverse Transcriptase Inhibitor). Hypertension (30\%) was the most common native kidney diagnosis followed by HIVAN (HIV associated Nephropathy), CIN (Chronic Interstitial Nephritis), DN (Diabetic Nephropathy) (20\% each) \& the least common was CGN (Chronic Glomerulonephritis) (10\%). One patient was HCV Ab (Hepatitis C Virus Antibody) positive and had to receive Peg interferon prior to transplantation, while none of the ten patients were positive for HBsAg (Hepatitis B Virus Surface Antigen). Prior approval was obtained from the Ethical committee of the hospital for the study.

To assess and evaluate the psychosocial impact of renal transplantation in these patients, a particular set of questions was prepared reflecting their psychological and social status. They were asked to fill this questionnaire after completion of at least 1 year of the renal transplant \& this included the impact on quality of life, relationship, psychological status, finances and overall outlook. We compared the pre and post-transplant states of these parameters wherever possible.

\section{Results}

Of the 10 HIV positive patients $9(90 \%)$ were males and $1(10 \%)$ was female.

\section{Questionnaire}

\section{Impact on quality of life}

Change in quality of life: Out of the ten, two (20\%) experienced better, eight $(80 \%)$ experienced much better quality of life. None reported a decline in the same post transplantation.

Ability to do job: Nine (90\%) patients answered that they were able to carry out their profession as usual. While one (10\%) patient was not able to resume his profession after the renal transplant (Figure 1).

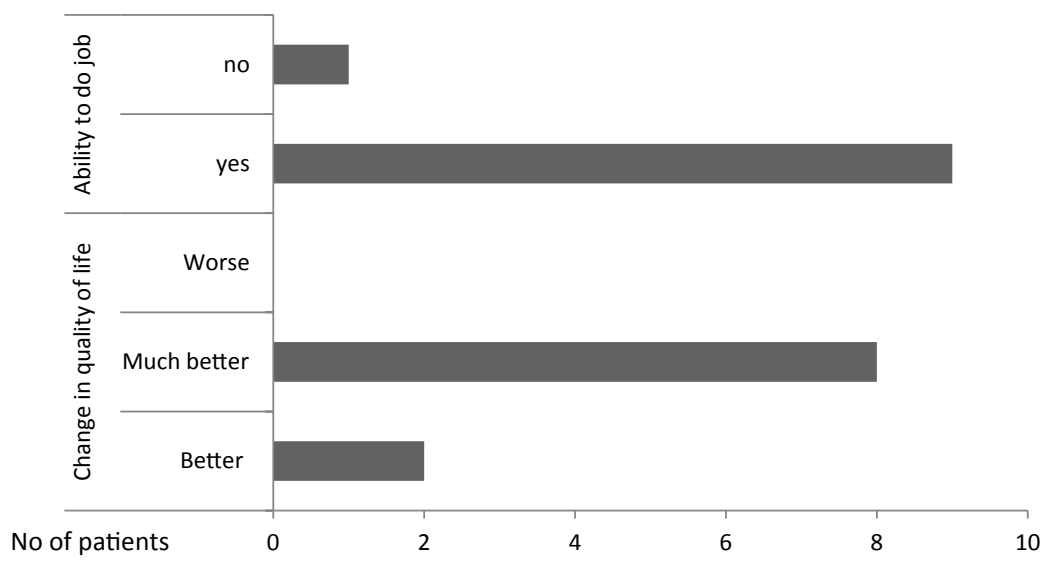

Figure 1: Impact of the renal transplant on the quality of life. 


\section{Impact on relationships}

Behavior of colleagues: Nine (90\%) patients reported experiencing a change in the behavior of their colleagues towards them post transplantation. Five (50\%) opined that it was better than before the renal transplant. Whereas the other four (40\%) described the change as "much better" than before.

Behavior of spouse: Out of the ten patients, four (40\%) patients experienced an improvement in the behavior of their spouse towards them. While five (50\%) felt that there was no appreciable change. The partner of one of the patients had died, thus the question did not apply for that patient.

Sexual relationship: Out of the 10 patients, four (40\%) perceived marked improvement in their sexual relationship, three (30\%) patients experienced minor improvement while two $(20 \%)$ did not feel any difference in their sexual relationship. The partner of one of the patients had died, thus the question did not apply for that patient. In conclusion, sexual relationship improved in 7 out of 9 patients (77\%) as one patient had lost their partner before the transplant (Figure 2).

\section{Impact on psychological status}

Fear of an unsuccessful transplant: Out of the 10 patients in the study, five (50\%) reported feeling anxious and feared an unsuccessful outcome, two $(20 \%)$ reported having had apprehensions to some degree ,while two (20\%) stated that they were not at all apprehensive of an unsuccessful outcome. Thus 70\% patients feared an unsuccessful outcome at the time of surgery.

Desire to live: Out of the 10 patients in the study, nine (90\%). Patients desired to live a longer life while one (10\%) patient did not desire to live a longer life.

Depression: Of the 10 patients, seven (70\%) reported not having faced depression at all, while two(20\%) patients faced some level of depression whereas one (10\%) of them had felt deeply depressed at some point post transplantation (Figure 3).

\section{Impact on treatment related problems}

Fear of relapse of HIV after transplant: Out of the 10 patients, six (60\%) feared the relapse of HIV while rest of the four (40\%) did not. Although none of the patients actually had a relapse till that time.

Immunosuppressive drugs calibration: Out of the 10, Seven (70\%) patients felt that they were frequently tested for calcineurin inhibitor drug levels and the doses were calibrated, while the remaining three (30\%) patients felt that they were tested infrequently.

Change in cART protocol: Out of the 10, the cART protocol of one (10\%) of the patients was altered. The remaining nine (90\%) patients did not require any variation in the cART protocol.

Increase in pill burden: After the transplant, all patients witnessed an increase in their pill burden due to addition of immunosuppressive drugs along with cART. Out of the 10 patients, nine (90\%) complained about the increase pill burden while one did not.

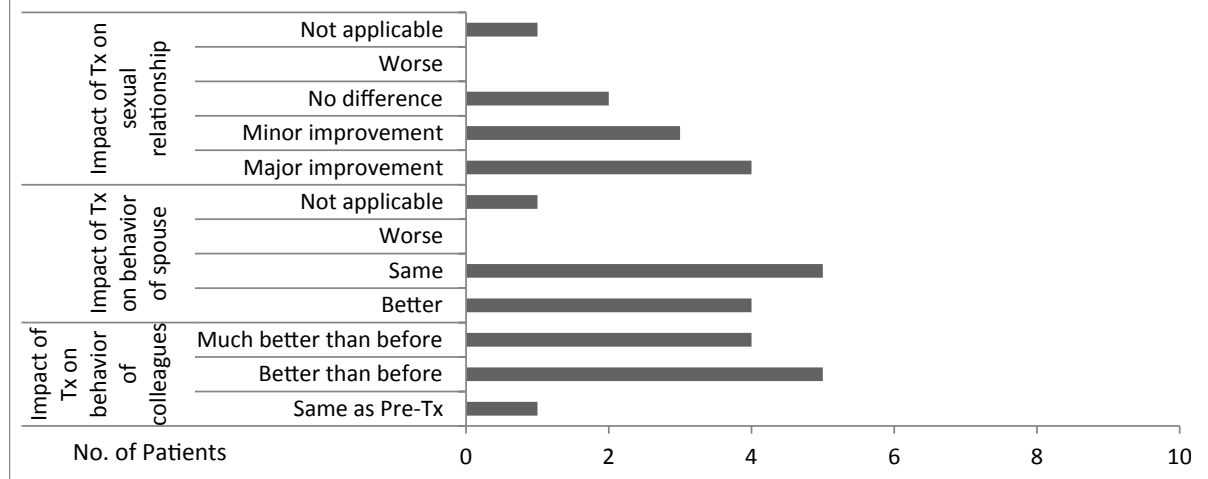

Figure 2: Impact of the renal transplant.. on relationship

Tx: Transplant

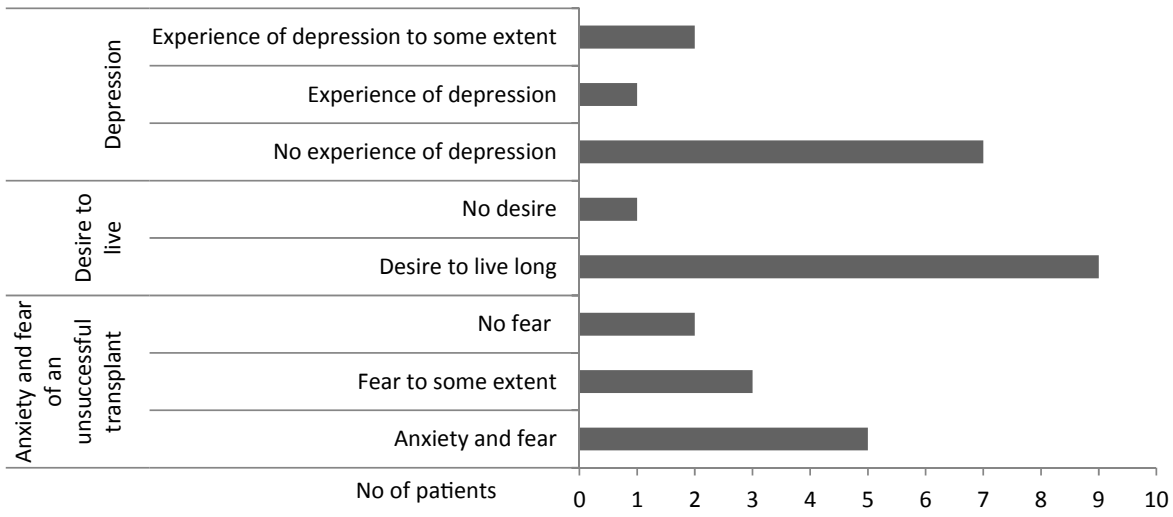

Figure 3: Impact on the psychological status. 


\section{Complications after Transplantation}

Of all the patients in the study, four (40\%) patients appeared to have signs of an infection, 2(20\%) had developed diabetes mellitus, $1(10 \%)$ was experiencing gastric problems and one (10\%) patient suffered from acute cellular rejection for which he received pulse corticosteroids. $5(50 \%)$ patients constantly feared an impending rejection while no complication was found post-transplantation in the remaining $2(20 \%)$ patients. There was no relapse of HIV in any of them.

\section{Preventive aspect and Impact on Financial Status}

\section{Cost factor}

Cost was considered as a major issue by all, however seven (70\%) were able to afford the surgery without too much difficulty whereas three (30\%) found it difficult to afford.

\section{Measures to prevent CKD in HIV patient}

Out of the 10 patients, $1(10 \%)$ patient conceptualized CKD as inevitable, $3(30 \%)$ patients suggested low viral load as effective in preventing CKD and 9 (90\%) patients believed that cART compliance is effective in preventing CKD.

$90 \%$ patients hypothesized cART compliance as a major factor in the prevention of CKD in HIV.

Impact on outlook towards renal transplantation as the modality of renal replacement therapy in HIV patients

Better modality of RRT: Among the 10 patients, Renal Transplantation was perceived to be the better method of RRT by nine (90\%) patients, one (10\%) patient believed that hemodialysis was better.

Elements restricting renal transplantation in ESRD HIV positive patients: Seven $(70 \%)$ patients opined that high cost and unawareness were the restricting elements.

Measures to enhance awareness about renal transplantation as an option in HIV positive patients with ESRD:Patients were asked about various measures to improve awareness. All the 10 patients were of the view that counseling of the patients at dialysis centres would be a great method to improve awareness. Additionally, 9 (90\%) patients felt that the family physician can help in increasing awareness.

\section{Discussion}

The study was aimed at determining the impact of renal transplantation on the psychosocial status of HIV positive patients. The study shows that all the HIV positive patients who underwent renal transplantation experienced better quality of life post transplantation. Of the 10 patients, nine were able to join and get back to their routine lives as before. There was an astounding improvement in the behaviour of the colleagues and spouses of the patients towards them post transplantation. One of the patients lost his partner, however seven patients experienced a radical change in their sexual relationships and were extremely cheerful post-transplant. The questionnaire showed that overall impact of renal transplantation on psychological status of the patients was indeed positive.

However, nearly $70 \%$ of the patients dreaded the failure of the renal transplant at the time of the surgery and about $60 \%$ patients had apprehensions of relapse of HIV infection due to use of immunosuppressive drugs. It should be noted that no one from the sample actually had a relapse of the infection. One of them did suffer from cellular rejection requiring pulse corticosteroids.
The patients felt that compliance with cART not only delays but also prevents CKD in HIV patients. Nearly $90 \%$ of the patients marked renal transplantation as the best modality of RRT which should be suggested to all the patients on dialysis therapy. Once the patient is approaching ESRD, they should be aware about renal transplantation as a possible option of RRT.

If we look for the components restricting renal transplantation in such patients; the gigantic cost of transplant takes the top spot. It may be unaffordable for some people with limited means. There is also lack of awareness about transplantation as an option in such patients. Awareness about renal transplantation can be expanded by informing patients at the dialysis centres. Counselling by the family physicians treating the HIV patients will also be very helpful. For this, they should be trained properly and informed that renal transplantation is a better modality of RRT in HIV positive ESRD patients.

These were found to be the best possible ways to make the transplant program successful for such patients. However further study on this topic with more number of patients is definitely required.

\section{Conclusion}

Patients who are known to have HIV infection, are on cART and have ESRD while being on maintenance hemodialysis face major trauma in their lives on psychosocial aspect. This study reveals that renal transplantation has drastically changed their lives and had a positive impact on their psychological status. Thus Renal transplantation should be encouraged for every HIV Positive patient with ESRD as a treatment option whenever feasible.

\section{Acknowledgment}

We acknowledge the help of the DNB registrars, residents, nursing staff, and OPD staff of our institute for the collection and management of the data used in this study. Abstract of this study was presented at $29^{\text {th }}$ OMICS conference in October 2014 held in the USA.

\section{References}

1. Ana-Maria Schweitzer, Michael B Mizwa, Michael W. Ross (2015) Psychosocial Aspects of HIVIAIDS: Adults

2. Rosenthal E, Poirée M, Pradier C, Perronne C, Salmon-Ceron D, et al. (2003) Mortality due to hepatitis C-related liver disease in HIV-infected patients in France (Mortavic 2001 study). AIDS 17: 1803-1809.

3. Rodriguez RA, Mendelson M, O'Hare AM, Hsu LC, Schoenfeld P (2003) Determinants of survival among HIV-infected chronic dialysis patients. J Am Soc Nephrol 14: 1307-1313.

4. EBPG (European expert group on renal transplantation) (2000) Evaluation selection and preparation of the potential transplant recipient. Nephrol Dial Transplant 15: 3-38.

5. Spital A (1998) Should all human immunodeficiency virus-infected patients with end-stage renal disease be excluded from transplantation? The views of US Transplant Centres 65: 1187-1191.

6. Kumar MS, Khan SM, Ranganna K, Malat GE, Kumar AS, Jacobson JM (2008) In HIV + patients with end stage renal disease (ESRD) kidney transplantation significantly prolongs long-term patient survival compared to chronic dialysis treatment. AmJ Transplant 8: 177-336.

7. Pelletier SJ, Norman SP, Christensen LL, Stock PG, Port FK, et al. (2004) Review of transplantation in HIV patients during the HAART era. Clin Transpl.

8. Abbott KC, Swanson SJ, Agodoa LY, Kimmel PL (2004) Human immunodeficiency virus infection and kidney transplantation in the era of highly active antiretroviral therapy and modern immunosuppression. J Am SocNephrol 15: 1633-9.

9. Roland ME, Carlson LL, Frassetto LA, Stock PG (2006) Solid organ transplantation: referral, management, and outcomes in HIV-infected patients. AIDS Read 16: 664-668, 675-8. 